\title{
Effects of aging on regular and express latencies of vergence
}

\author{
Qing Yang \\ Iris Laboratory, FRE 3154, CNRS
}

\author{
Thanh-Thuan Lê \\ Iris Laboratory, FRE 3154, \\ CNRS
}

\author{
Zoi Kapoula \\ Iris Laboratory, FRE 3154, \\ CNRS
}

\begin{abstract}
Vergence eye movements are frequent in every day life and important for depth perception. Yet, studies of vergence in elderly are rare. We examined convergence and divergence between targets placed along median line at 20,40 or $150 \mathrm{~cm}$. Thirteen elderly ( $70 \pm 11$ years) and ten young ( $25 \pm 3$ years) adults participated in the study. The gap paradigm (i.e., the fixed stimulus is extinguished prior to target onset) and the overlap paradigm (the fixed stimulus remains illuminated after target onset) were used to elicit reflexive or voluntary eye movements. Latency of convergence and divergence increased with age in both gap and overlap conditions. Both young subjects and elderly showed shorter latency of vergence under the gap condition than under the overlap condition. In the overlap condition, for elderly only, convergence resulted in longer latencies than divergence. In the gap condition express convergence was rare, while express latencies (80-120ms) were frequent for divergence starting from a near fixation point (at $20 \mathrm{~cm}$ ). The rates of express divergence were similar for young (23\%) and elderly $(21 \%)$. These results were in line with prior studies of saccades and indicated different aging effects on regular and express latencies.
\end{abstract}

Keywords: Elderly, Convergence, Divergence, Regular latency, Express latency

\section{Introduction}

Fine Both stereopsis and bifoveal fixation of object of interest require precise alignment of the visual axes, which depend on vergence eye movements (Leigh \& Zee, 2006). Vergence eye movements are necessary to change fixation from a far to a near target (convergence), or vice versa (divergence). The initiation of vergence eye movements has been studied at the early 1970's; most of these studies concerned the disparity driven vergence movements, e.g. by use of prisms. The latency for a disparitydriven vergence movement is about $160 \mathrm{~ms}$ (Leigh \& Zee, 2006). More recent studies concerned vergence eye movements with targets in natural 3D space for which both disparity, accommodation and other high level proximal cues are involved (Kumar, Han, Garbutt \& Leigh, 2002; Rambold, Neumann, Sander \& Helmchen, 2006;
Yang, Bucci \& Kapoula, 2002). Latency of such vergence is about $200 \mathrm{~ms}$. For young adults, there is difference between latencies of convergence and divergence (Alvarez, Semmlow \& Pedrono, 2005; Hung, Zhu \& Ciuffreda, 1997; Krishnan, Farazian \& Stark, 1973; Semmlow \& Wetzel, 1979; Yang et al., 2002). For elderly, only one study (Rambold et al., 2006) reported no latency difference between convergence and divergence, which needs further investigation.

Here we are also interested in the role of attention on vergence latency for young and elderly adults. The latency of eye movements can be influenced by the temporal 
relationship between the offset of the fixation point and the onset of the target (Saslow, 1967). Latency is decreased in the gap paradigm (fixation light turns off before the onset of the target), i.e. gap effect. This was first confirmed for saccades by Saslow (1967), then extended to anti-saccades (Fischer \& Weber, 1997; Goldring \& Fischer, 1997), to smooth pursuit (de Hemptinne, Lefevre \& Missal, 2006; Knox, Davidson \& Anderson, 2005), and to vergence (Bucci, Pouvreau, Yang \& Kapoula, 2005; Coubard, Daunys \& Kapoula, 2004; Takagi, Frohman \& Zee, 1995; Tam \& Ono, 1994). For saccades, such gap effect shows both general decrease of regular latencies between 150 and 400 ms (Fischer, Weber, Biscaldi, Aiple, Otto \& Stuhr, 1993; Weber \& Fischer, 1995) and increase of the rates of express latencies between 80 and 120 ms (Fischer et al., 1993; Munoz, Broughton, Goldring \& Armstrong, 1998). For both convergence and divergence the gap paradigm reduces similarly the mean latency (Coubard et al., 2004; Takagi et al., 1995). However, the rates of express latencies are higher for divergence than for convergence (Bucci et al., 2005; Coubard et al., 2004; Kapoula, Isotalo, Muri, Bucci \& RivaudPechoux, 2001).

It should be noted that knowledge of the cortical and subcortical substrate of vergence is continuously increasing in the recent years. For example, in FEF there are neurons that discharge for convergence or divergence eye movements made in response to step displacement of a target in depth (Gamlin \& Yoon, 2000). Some neurons in the parietal lobe were found to encode retinal disparity that drives changes of fixation in depth (Genovesio \& Ferraina, 2004). Transcranial magnetic stimulation (TMS) over the right PPC increased the latency for vergence movements (Kapoula et al., 2001; Kapoula, Yang, Coubard, Daunys \& Orssaud, 2005a; Kapoula, Yang, Coubard, Daunys \& Orssaud, 2005b) while TMS over DLPC decreased the latency of convergence movements (Coubard \& Kapoula, 2006). In midbrain, vergence burst cells display a discrete burst of activity just before and during vergence eye movements (Mays, Porter, Gamlin $\&$ Tello, 1986)). There is evidence in a patient (Ohtsuka, Maeda \& Oguri, 2002) or in cats (Suzuki, Suzuki \& Ohtsuka, 2004) that the rostral superior colliculus is also involved in the control of vergence. Unlike saccadic eye movements (Abel, Troost \& Dell'Osso, 1983; Huaman \& Sharpe, 1993; Irving, Steinbach, Lillakas, Babu \& Hutch- ings, 2006; Munoz et al., 1998; Yang et al., 2002; Yang, Kapoula, Debay, Coubard, Orssaud \& Samson, 2006), to our knowledge, the only study with objective measures of vergence eye movements in healthy elderly is that of Rambold et al. (2006).

The present study aims to explore aging effects on convergence and divergence latency in two tasks (gap and overlap); vergence movements start from different initial positions $(20 \mathrm{~cm}$ and $40 \mathrm{~cm}$ for divergence, $40 \mathrm{~cm}$ and $150 \mathrm{~cm}$ for convergence). The study aims to provide a more complete view of vergence performance in $3 \mathrm{D}$ space with age and to understand aging effects for different neurophysiologic mechanisms and circuits controlling such eye movements. Based on prior work on saccades (Yang et al., 2006) we expected latencies of vergence to be longer for elderly. However, to what extend express type of vergence can still be possible in elderly is not known. As in the past we expected different aging effects on different types of latencies for vergence.

\section{Methods and Materials}

\section{Subjects}

Thirteen subjects aged between 60 and 93 years

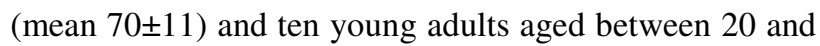
32 years (mean 25 \pm 3 ) participated in the study. All young subjects were students from the laboratory and had normal visual acuity. Elderly subjects were recruited either among senior laboratory workers, or in the ophthalmology service in the hospital, coming for routine examination and spectacle correction or at sport centers for elderly in the proximity of the laboratory. They had corrected visual acuity more than $5 / 10$, i.e. normal for their age. The cognitive performance as measured by Mini Mental State Examination (MMSE, was better than 26 of 30 points for elderly subjects. No subject showed visual, neurological, psychiatric disorders or received medication. For both groups binocular vision was assessed with the test of stereoacuity (the Netherlands Organization of Applied Scientific Research); all individual scores were normal, $60 "$ of arc or better. The investigation adhered to the tenets of the Declaration of Helsinki and was approved by the institutional human experimentation committee. Informed consent was obtained from all subjects after the nature of the procedure had been explained. 


\section{Visual display}

The visual display on a horizontal table consisted of LEDs (each LED on $2.9 \mathrm{~mm}$ ) placed at three viewing distance in middle line, one at $20 \mathrm{~cm}$ from the subject, and the others at $40 \mathrm{~cm}$ and $150 \mathrm{~cm}$. Fixation to one of three LEDs at center requires vergence angle of $17.1^{\circ}$, $8.6^{\circ}$ and $2.3^{\circ}$ for targets at 20,40 and $150 \mathrm{~cm}$, respectively (Figure 1A). In a dark room, subject was seated in an adapted chair with a chin and frontal rest. $\mathrm{He} /$ she viewed binocularly and faced the visual display of the LEDs. Vertically, all target LEDs were placed at eye level.

\section{Fixation and oculomotor tasks: gap and overlap conditions}

Each trial started by lighting a fixation LED at the center. The fixation LED stayed on for a random period between 1.5 and $2 \mathrm{sec}$. In the gap task, there was a time interval of $200 \mathrm{~ms}$ between the offset of the fixation point and the onset of the vergence target. The target LED was kept on for $1.5 \mathrm{sec}$ (Figure 1B). In the overlap task, the fixation point remained illuminated for $200 \mathrm{~ms}$ after the target LED appeared. The target stayed on also for another $1.5 \mathrm{sec}$ (Figure 1C). Subjects were required to make a vergence to the other central target LED as rapidly and accurately as possible. A black period of 500ms separated trials. Subjects were instructed to use this period for blinks. The total mean length of each trial was about 4 sec. In each block divergence (from $20 \mathrm{~cm}$ to $40 \mathrm{~cm}$ or from $40 \mathrm{~cm}$ to $150 \mathrm{~cm}$ ) and convergence (from 150 to 40 $\mathrm{cm}$ or from $40 \mathrm{~cm}$ to $20 \mathrm{~cm}$ ) were interleaved randomly at equal rates. Each block contained 60 trials and lasted 4 min. each subject performed two blocks, one in the gap condition and another in the overlap condition.

A calibration sequence was performed at the beginning and at the end of each block; the target made the following predictive sequence for each viewing distance: center, $5^{\circ}$ to left, center, $10^{\circ}$ to left, center, $5^{\circ}$ to right, center, $10^{\circ}$ to right, center; the target stayed at each location for $2 \mathrm{sec}$. From these recordings we extracted calibration factors.

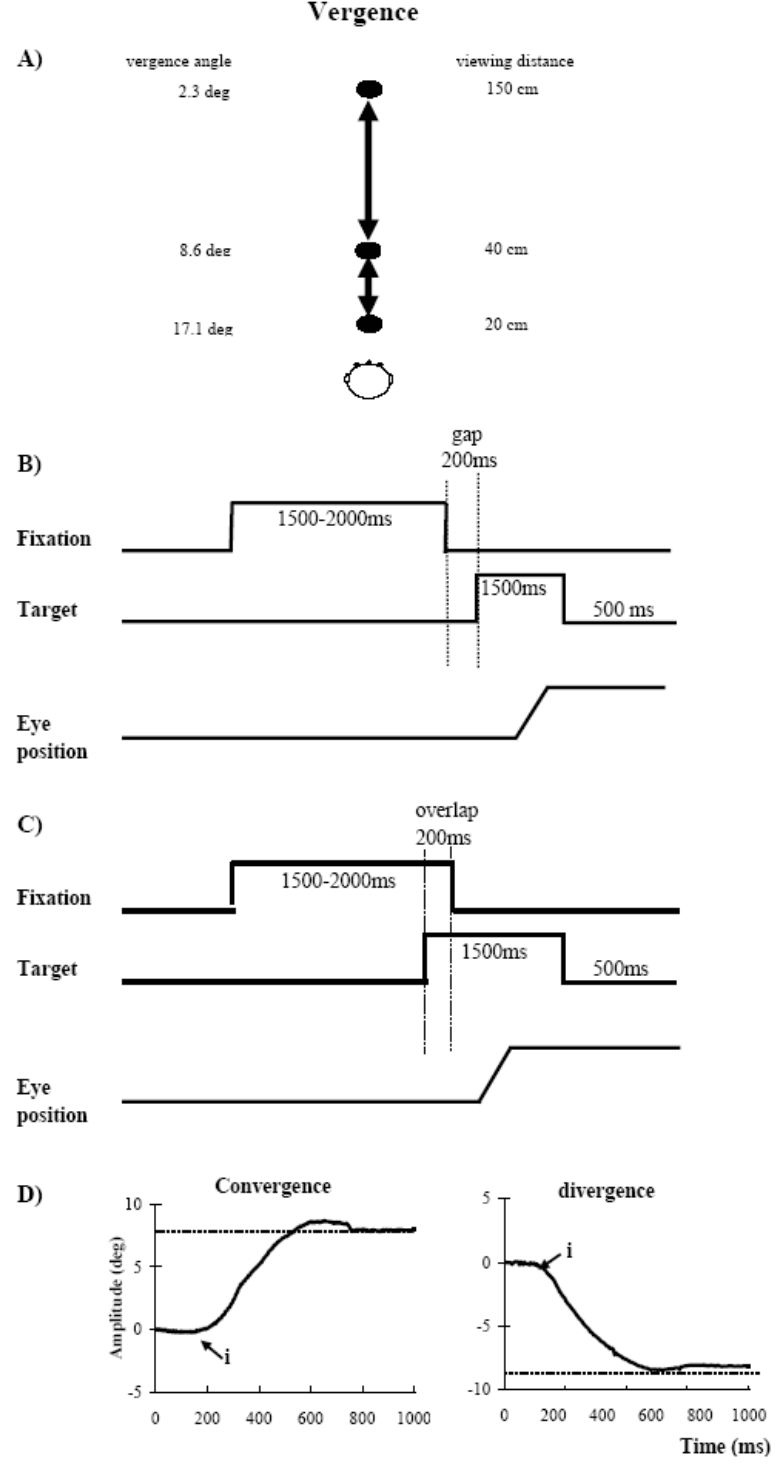

Figure 1. (A) Three LEDs (light emitting diodes) along median line were place on the horizontal table at $20 \mathrm{~cm}, 40 \mathrm{~cm}$ and $150 \mathrm{~cm}$ in front of subject for eliciting convergence and divergence. (B) The paradigm used for the gap condition, the central fixation point disappears $200 \mathrm{~ms}$ before the appearance of the eccentric target. (C) For the overlap condition, the central fixation point remains illuminated when an eccentric target appears. (D) Typical recordings of vergence are obtained by difference of the position signal of the two eyes LE-RE. The arrows at ' $i$ ' indicate the onset of vegence. 


\section{Eye movement recording}

Horizontal movements from both eyes were recorded simultaneously with the IRIS, SKALAR device for six young and four elderly subjects. Eye position signals were low-pass filtered with a cut-off-frequency of $200 \mathrm{~Hz}$ and digitized with a 12-bit analogue-to-digital converter and each channel was sampled at $500 \mathrm{~Hz}$. For the other four young adults and nine elderly subjects, eye movements were recorded with the Chronos rapid video eye tracker, which is based on high-frame rate CMOS sensors (Clarke, Ditterich, Druen, Schonfeld \& Steineke, 2002). With the video eye tracker, eye position data were sampled at $200 \mathrm{~Hz}$ and stored on the hard disk for off-line analysis. This system allowed recording of both horizontal and vertical components while the IRIS system allowed recording of horizontal components only. Nevertheless, by placing the LED table at the eye level, vertical eye movements were minimized for all experiments. The head was always stabilized by a chinrest and/or a forehead rest.

\section{Data analysis}

From the two individual calibrated eye position signals we derived the disconjugate signal (left eye - right eye). The onset of the vergence eye movements was defined as the time point when the vergence velocity exceeds $5 \%$ s (Figure 1D, point ' $i$ '). This criterion is standard (Takagi et al. 1995; Yang et al. 2002). The process was performed automatically by the computer, and the verification was made by visual inspection of the individual eye position and velocity trace.

For both convergence and divergence, we measured the latency, i.e. the time between target onset $(0 \mathrm{~ms})$ and vergence onset (marker ' $i$ ' in Figure 1D), and we also evaluated the percentage of express type of short latency (80-120ms).

Eye movements in the wrong direction, with latency shorter than $80 \mathrm{~ms}$ (anticipation) or longer than $800 \mathrm{~ms}$, or contaminated by blinks were rejected. For adults seven percent of trials and for elderly subjects nine percent of trials had to be rejected using these criteria.

A multiple analysis of variance (ANOVA) was performed on individual mean values of each parameter with the factor between subjects - the age (young, elderly), and the factors within subjects - the oculomotor task (gap, overlap), the starting point (near, far) and vergence (convergence, divergence). Post-hoc comparisons were done with the Least Significant Differences test. For the percentages of express vergence, the non-parametric test Wilcoxon test was used for within-subjects comparisons between different conditions; the U-Mann-Whitney test was used for comparisons between young and elderly subjects.

\section{Results}

\section{Individual and group latencies}

The individual mean latencies of vergence and the standard errors (SE) were shown for the gap and the overlap conditions in adults and elderly in Figure 2 (convergence starting from $40 \mathrm{~cm}$ and divergence from 20 $\mathrm{cm}$ ) and Figure 3 (convergence starting from $150 \mathrm{~cm}$ and divergence from $40 \mathrm{~cm}$ ). With a few exceptions indicated by arrows (in Fig.2 and 3) all subjects showed shorter latency in the gap condition than in the overlap condition.

The multiple ANOVA applied on the latency values with showed the following main effects: a significant effect of age $\left(F_{1,21}=11.31, p<0.01\right)$, i.e. longer latencies for elderly than for young subjects; a significant effect of gap task $\left(\mathrm{F}_{1,21}=53.85, \mathrm{p}<0.001\right)$, i.e. shorter latency in the gap than in the overlap condition; a significant effect of type of vergence $\left(F_{1,21}=7.81, p<0.01\right)$, i.e. longer latencies for convergence than for divergence, but no effect of starting point $\left(\mathrm{F}_{1,21}=3.94, \mathrm{p}>0.05\right)$. All significant effects are summarized in Figure 4 (A, B and C).

A significant interaction was found between task and vergence $\left(F_{1,21}=7.14, p<0.01\right)$, i.e. longer latency for convergence than for divergence in the overlap condition only (Figure 4D). Further post-hoc comparisons showed that the age effect was significant in all conditions (all $\mathrm{p}<0.05$ ) except for divergence starting from near (at $20 \mathrm{~cm})$ in the gap condition $(\mathrm{p}=0.29)$. The gap effect was significant for all conditions in both young and elderly adults (all $\mathrm{p}<0.01)$. The vergence effect was significant in the overlap condition in elderly only $(\mathrm{p}<0.001$ for vergence starting from near, $\mathrm{p}<0.05$ for vergence starting from far point). 

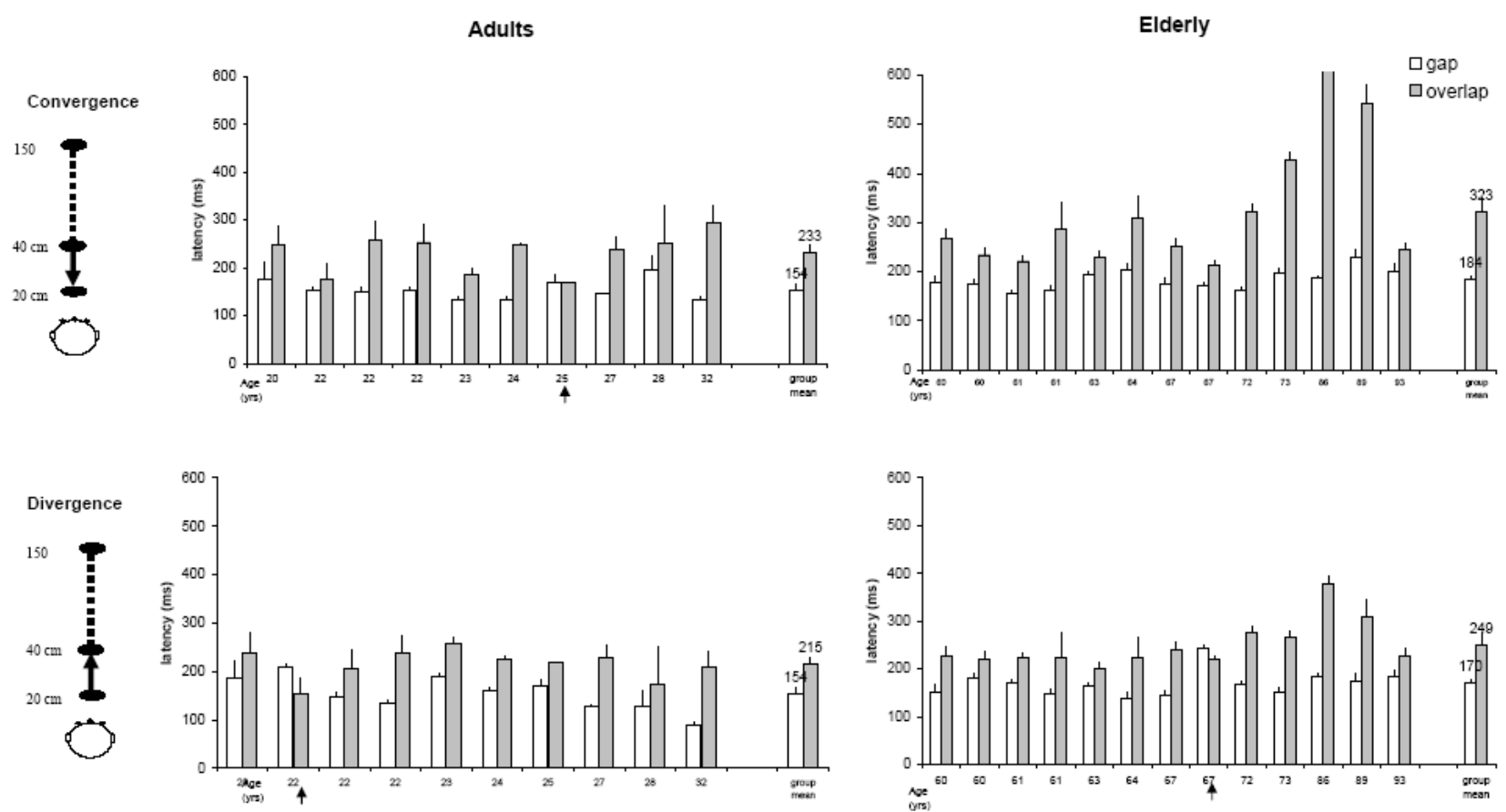

Figure 2. Individual and group mean latency with standard errors for convergence or divergence starting from near fixation point (two LEDs at $20 \mathrm{~cm}$ and $40 \mathrm{~cm}$ in front of subject, respectively) in the gap and the overlap conditions for both young and elderly subjects.
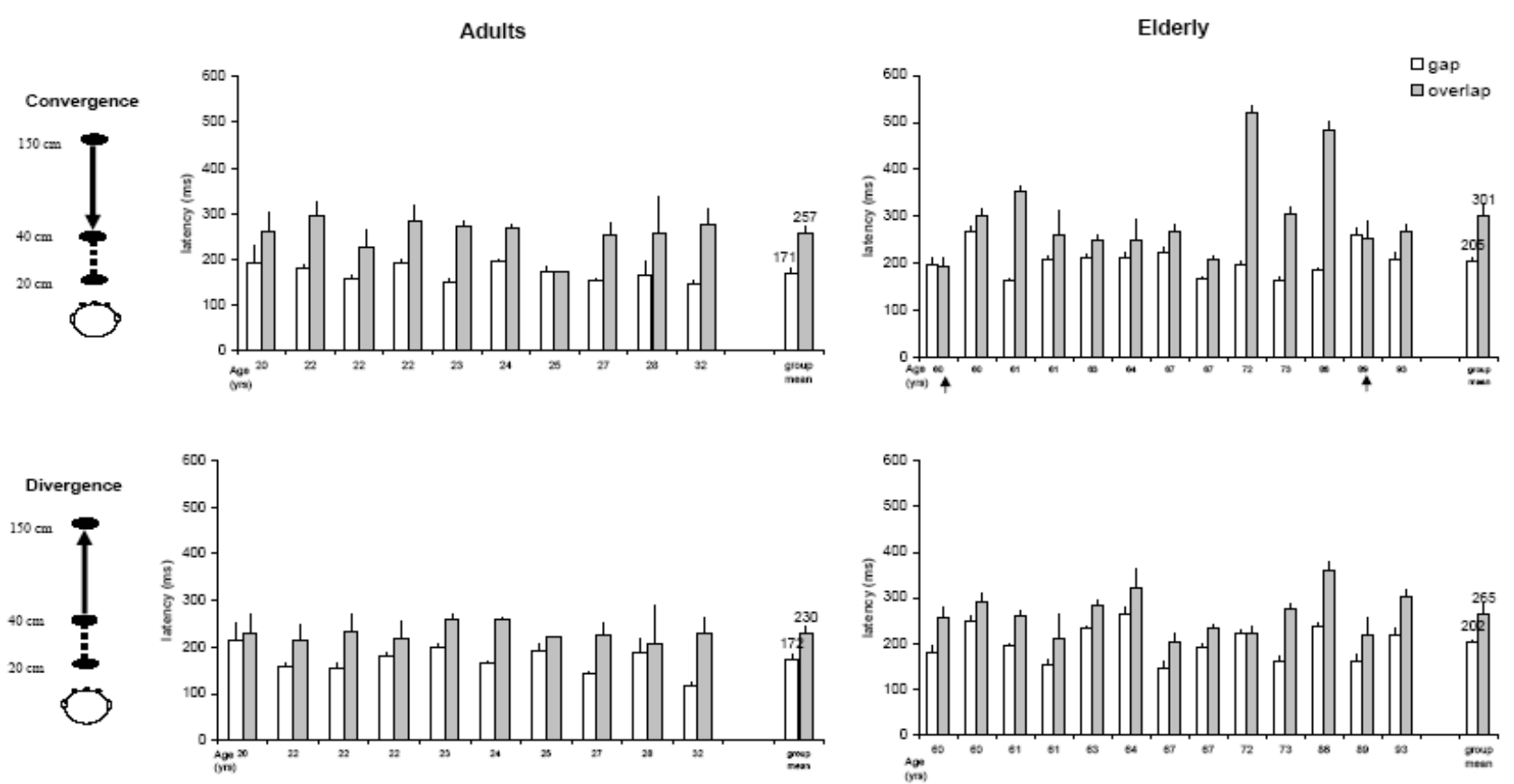

Figure 3. Individual and group mean latency with standard errors for convergence or divergence starting from far fixation point (two LEDs at $40 \mathrm{~cm}$ and $150 \mathrm{~cm}$ in front of subject, respectively) in the gap and the overlap conditions for both young and elderly subjects. 
A

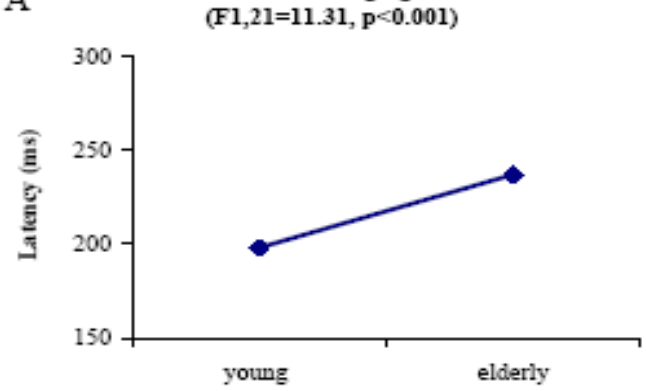

B

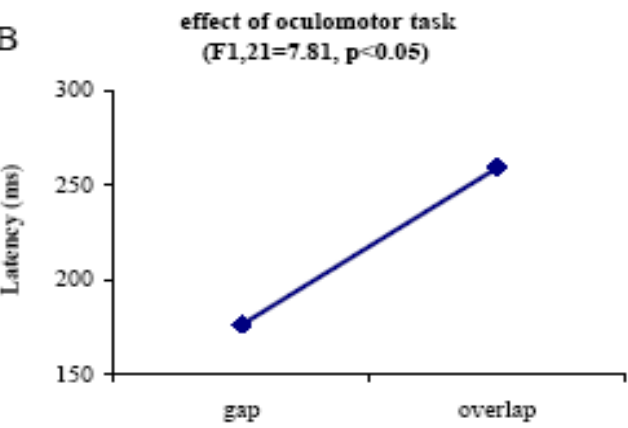

$\mathrm{C}$
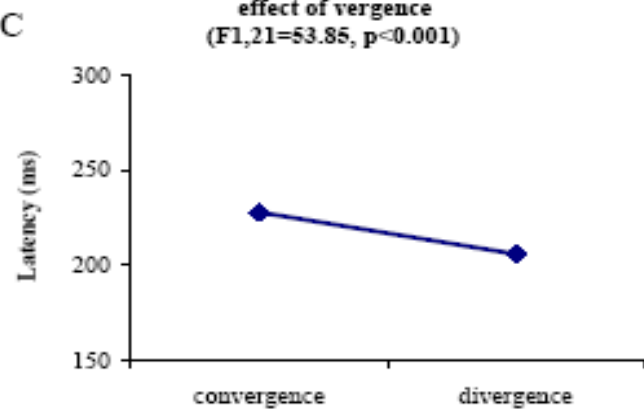

$\mathrm{D}$

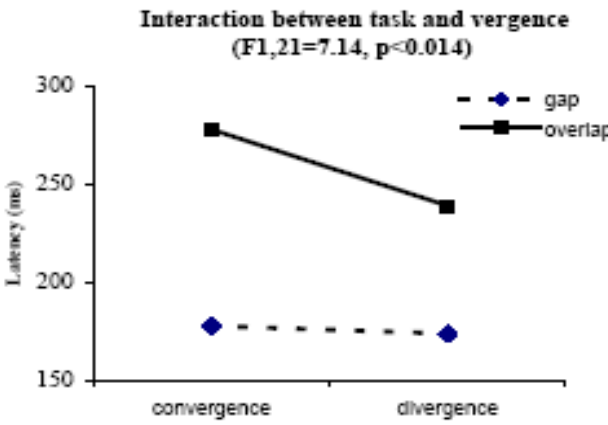

Figure 4. Main effects of the age (A), of the gap (B), of the vergence $(C)$, and interaction between task and vergence (D). Vertical lines indicate the standard errors. Asterisks indicate statistically significant effects.
As mentioned in Introduction, there are different types of latencies of saccades (Fischer \& Weber, 1997; Gezeck \& Timmer, 1998): express type (80-120ms), fast regular (121-150ms) and slow regular (151-400ms), which were used for vergence here also. A second ANOVA analysis was done on mean latency values excluding express type of latencies (these will be further presented later). Because one young subject produced $100 \%$ express type near divergence in the gap condition, analysis on mean latencies without express latency was made on nine young adults and thirteen elderly subjects. The ANOVA showed again significant effects of age $\left(\mathrm{F}_{1,16}=12.10, \mathrm{p}<0.01\right)$, and of gap task $\left(\mathrm{F}_{1,16}=38.13\right.$, $\mathrm{p}<0.0001)$. Further post-hoc comparisons showed that the age effect was significant for all conditions (all $\mathrm{p}<0.05$ ) including for divergence starting from near. Thus, it is the regular type of latency of vergence which is slowed by aging.

\section{Express latencies}

Express latencies are between $80-120 \mathrm{~ms}$ as established by analogy with express saccades. In the gap condition 6 of 10 young adults and 8 of 13 elderly subjects produced a substantial number of express type of vergence (ranging between $0 \%$ and $100 \%$ ). The mean percentages were $4 \%, 23 \%, 0 \%$ and $7 \%$ for convergence or divergence starting from near, and for convergence or divergence starting from far in young subjects; the corresponding values in elderly were $0 \%, 21 \%, 5 \%$ and $8 \%$ (Figure 5). The Mann-Whitney U test showed no significant difference of the percentage of express vergence between young and elderly (all $\mathrm{p}>0.05$ ). The Friedman test showed significantly different percentage of express latencies among different type of movements in both young $\left(\mathrm{F}_{3,10}=10.11, \mathrm{p}<0.05\right)$ and elderly $\mathrm{F}_{3,13}=12.1$, $\mathrm{p}<0.01)$. Further Wilcoxon test showed statistically higher percentage for divergence starting from near than for other types of vergence in both young and elderly subjects (all $\mathrm{Z}>1.9, \mathrm{p}<0.05$ ). There was no statistically significant difference among other types of vergence (divergence starting from near, convergence starting from near or far), neither in young nor in elderly subjects (all $\mathrm{Z}<1.8$, $\mathrm{p}>0.05)$. Under overlap condition express vergence was rare; only two elderly produced express latencies (1 or 2 trials, respectively) for divergence starting from near. 
Thus, express type latency occurring specifically for divergence is not affected by aging.

\section{Gap paradigm}

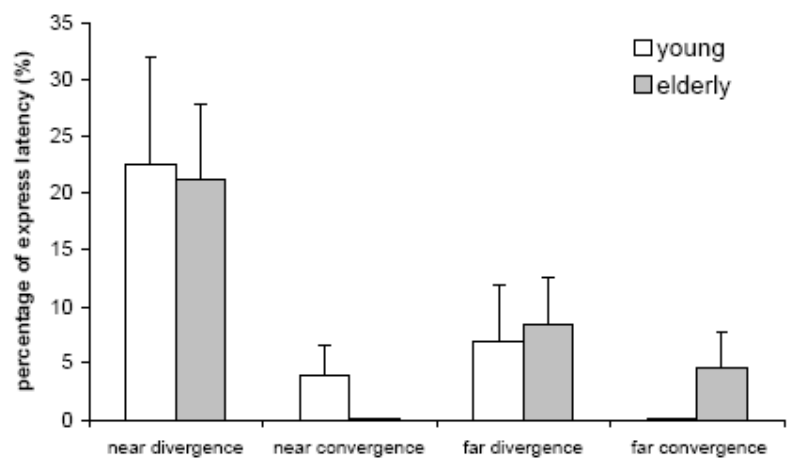

Figure 5. Percentages of express latencies of convergence and divergence for both young and elderly subjects. Near divergence starting from point at $20 \mathrm{~cm}$; near convergence starting from point at $40 \mathrm{~cm}$; far divergence starting from point at 40 $\mathrm{cm}$; far convergence starting from point at $150 \mathrm{~cm}$. Percentages of express latency of near divergence are significantly higher than those of other type of vergence for both young and elderly subjects (both $\mathrm{p}<0.05$ ).

\section{Discussion}

The main findings of this study are: 1) Regular latencies of vergence are longer for elderly than for young subjects in both gap and overlap conditions. 2) Like young subjects, elderly, show shorter latency of vergence under the gap condition than under the overlap condition (gap effect). 3) Convergence shows longer latencies than divergence in the overlap condition for elderly only. 4) Express type of latencies are frequent under the gap condition, particularly for divergence starting from near; this is the case for both young and elderly subjects.

\section{Increase of regular vergence latency in elderly}

The only prior study of latency of vergence in elderly using simultaneous offset of fixation and onset of vergence target showed an age-related increase in latency (Rambold et al., 2006). Our data extend this observation to both gap and overlap conditions and provide a new insight for the aging effect on regular versus express type of latency. Indeed, elderly showed longer regular latencies of vergence under both gap and overlap conditions than young subjects. Slowing of the motor reaction time is a well-documented general phenomenon with aging (Katzman \& Terry, 1983; Rambold et al., 2006). The present results are in line with our prior studies of saccades (Yang \& Kapoula, 2006; Yang et al., 2006), and all show that elderly had longer latency for both types of eye movements, saccades and vergence. The increase of latencies of eye movements could be related to the increased processing time at any or all stages from sensory process of stimulus to the eye-movement output (Knox et al., 2005).

Several hypothetical processes are involved in the control of latency of saccades and vergence, e.g. release of ocular fixation, shift of visual attention, computation of the metrics of the eye movements, and decision to move the eyes. Such hypothetic processes take place in the cortical areas including frontal and parietal cortexes (Leigh \& Zee, 2006); the role of such areas on eyemovement control had been confirmed by many studies in patients with cortical lesions (Pierrot-Deiseilligny et al. 2003, 2005), in monkeys with cortical ablation (Gamlin \& Yoon, 2000; Schiller, Sandell \& Maunsell, 1987), and in normal subjects by use of TMS (Kapoula et al., 2001; Kapoula et al., 2005a; Kapoula et al., 2005b; Muri, Vermersch, Rivaud, Gaymard \& Pierrot-Deseilligny, 1996; Yang \& Kapoula, 2004). Physiological brain aging is characterized by a loss of synaptic contacts and neuronal apoptosis that provokes aging-dependant decline of sensory processing, motor performance and cognitive function (Rossini, Rossi, Babiloni \& Polich, 2007), which could slow the reaction time of eye movements. During normal aging, histological analysis showed widely spread atrophy in cerebral cortex including both gray and white matter (Creasey \& Rapoport, 1985). Magnetic resonance imaging (MRI) studies confirmed that both the frontal lobe and parietal cortex underwent progressively degenerative changes with aging in humans (Head, Buckner, Shimony, Williams, Akbudak, Conturo, McAvoy, Morris \& Snyder, 2004; Salat, Kaye \& Janowsky, 2001). Superior colliculus and brainstem oculomotor generator could also be related to the processes of latency although histological studies have failed so far to detect age-related neuronal losses in these structures (Henson, Staunton \& Brett, 2003; Katzman \& Terry, 1983; Vijayashankar \& Brody, 1977a; Vijayashankar \& Brody, 1977b). 
Another possible explanation of the prolongation of eye-movement reaction time in elderly could be deliberate adoption of a slower global mode of functioning, e.g. allowing for extra time to attend better where to look next. This idea is in line with theoretical studies that have suggested that aging would not be due to local errors, but would be inherent to the global organization of a complex system (Rosen, 1978), in which the local and global systems can influence each other. To summarize, the slow latency of vergence in elderly could be related to hypofunction of cerebral oculomotor areas, and/or to adoption of general slower mode of functioning. However, the results on express type of latency discussed next go against the global theory as they indicate the existence of specific mechanisms and differential aging effects.

\section{Gap effect}

For gap effects, latency decreases in the gap task relative to non-gap task, and express latency have been widely studied for saccades but for vergence. We report that healthy elderly also show a gap effect for vergence latencies and are capable of producing express type of latency for vergence. Moreover, the percentages of reduction of regular vergence latency under the gap condition relative to the overlap condition were similar in young (30\%) and elderly subjects (33\%). The results are comparable to those we reported previously for horizontal saccades (35\% vs $32 \%$ ) and vertical saccades (29\% vs 27\%). The gap effect on regular saccade latency has been attributed to various hypothetical mechanisms: the offset of fixation prior to target onset could act as warning signal facilitating the disengagement of fixation and motor system preparation thereby shortening the reaction time of eye movements (Kingstone \& Klein, 1993; Reuter-Lorenz, Hughes \& Fendrich, 1991; Ross \& Ross, 1980; Ross \& Ross, 1981). This gap effect may be the result of several factors (Csibra, Johnson \& Tucker, 1997), including the disengagement of visual fixation prior to target onset (Munoz et al., 1998). In the overlap task, fixation is actively engaged at the time of target presentation and its disengagement requires volitional control leading to the increase of latencies. According to the recent model of Findlay and walker (Findlay \& Walker, 1999) during the gap duration the activity in the fixate centre is decreased because of the absence of stimulus (neither fixation nor target) and thereby saccade triggering is enhanced. All these hypothetical mechanisms for the gap effect could be similar for saccades as well as for vergence. Thus, our results suggest that elderly, similarly to young adults, can benefit from early offset of visual fixation and thereby generating shorter latency of vergence.

\section{Express divergence}

Another important result is that elderly can produce frequently express latencies of vergence, especially for divergence starting from near, in the gap condition. Note that the rates of such latencies in elderly are similar to those in young adults (21\% vs $23 \%$ for divergence starting from near; $9.2 \mathrm{vs} 8.6 \%$ for divergence starting from far, for elderly and young adults, respectively). In contrast, both young and elderly subjects have very few cases of express type of convergence. What are the specific mechanisms facilitating express type of divergence? The relaxation of convergence from near, or the warning and disengagement of attention because of the offset of fixation or the predictability of new target could be involved in promoting express divergence latencies. Yang et al. (2006) showed that in the gap condition when fixating at near viewing distance $(20 \mathrm{~cm})$ express latencies for horizontal saccades are more frequent compared to when fixating at far viewing distance for both young (23\% vs $9 \%$ ) and elderly (21\% vs 1\%). Divergence in the present study induces always relaxation of convergence. In general, when divergence starts from near point many factors could contribute to shorten latency, e.g. fixation offset (gap), attention disengagement of ocular fixation, relaxation of convergence and prediction of divergence

\section{Models of express latency}

Isa and Kobayashi (2004) emphasize that express saccades are mediated by 'short-loop' brainstem pathway (retina - SC - extraocular muscles). Other investigators proposed that cortical areas, such as occipital cortex (Schiller \& Tehovnik, 2005), parietal posterior cortex (Fischer \& Weber, 1993; Pierrot-Deseilligny, Muri, Ploner, Gaymard \& Rivaud-Pechoux, 2003) and frontal eye field (Brown, Bullock \& Grossberg, 2004) are also involved in the express-loop. No matter which neural circuit is involved in the control of express latency, we suggest that the circuit is similar for express saccades and express vergence; this is in line with a TMS study by Coubard and Kapoula (2006), showing high express la- 
tencies for both vergence and saccades after interference of the dorslateral prefrontal cortex by TMS.

Here we will emphasize that express latencies correspond to minimal afferent and efferent conduction (Carpenter, 1981; Munoz et al., 1998). Their occurrence in elderly similarly to young subjects indicates that there is no downstream difficultly to generate express-type latencies with age. Such result is also in line with the idea of 'short-loop' for producing express saccades and perhaps also for express vergence (Isa \& Kobayashi, 2004). Note that stimulation in the rostral pole of superior colliculus can induce vergence eye movements and the activity of rostral neurons can be modulated by pure vergence (Chaturvedi \& Van Gisbergen, 2000). Moreover, there is evidence suggesting no degeneration of brainstem structures in general with aging (Henson et al., 2003; Katzman \& Terry, 1983; Vijayashankar \& Brody, 1977a; Vijayashankar \& Brody, 1977b). Finally, it is possible for a complex system to exhibit global modes of failure which are not associated with local subsystem failure (Rosen, 1978). The capacity to generate express type of eye movements in 3D space is an example at such local subsystem preserved. The fact that express divergence latency was more frequent for divergence starting from near $(20 \mathrm{~cm})$ than for divergence starting from far $(40$ $\mathrm{cm})$ suggests that convergence relaxation is indeed one of the factors contributing to such short latency, together with a warning effect and with target predictability. Indeed, when the fixation point was present at $20 \mathrm{~cm}$ the only possible target location was at $40 \mathrm{~cm}$, i.e. calling for divergence. The present study does not allow to distinguish the relative importance of convergence relaxation versus target predictability for triggering express divergence.

\section{Convergence vs divergence latency}

Latency of convergence was longer than that of divergence for elderly under the overlap condition only. For young subjects, reports in the literature are contradictory in the latency between convergence and divergence. Using targets in natural 3D space, Rambold et al. (2005) reported that there was no the difference of latency between convergence and divergence in subjects in the age range of 19-73 years; Semmlow \& Wetzel (1979) stated that convergence latency is less than divergence latency, while other researchers have reported the opposite, e.g. convergence has a longer latency than divergence (Krishnan et al., 1973; Yang et al., 2002). For disparity driven vergence, the results for latency difference between convergence and divergence are also contradictory (Alvarez et al., 2005; Hung et al., 1997; Rashbass \& Westheimer, 1961). Contradicting findings could be partially related to differences between individuals. Another factor influencing vergence latency is the initial starting position, particularly for divergence (Alvarez et al., 2005). In addition, different thresholds of velocity used by different authors to identify the onset of vergence could also influence the evaluation of such difference between convergence and divergence. The present study compares vergence latency between young and elderly subjects in gap and overlap tasks using the same criteria for determining the onset of vergence. The gap task promoting a rather reflexive mode of triggering of eye movements showed no differences in latency between convergence and divergence. In contrast, the overlap paradigm involved higher volitional control of fixation and attention disengagement and revealed difference in latency between convergence and divergence in elderly. Indeed, in the overlap condition, for 11 out of 13 elderly subjects the latency differences between convergence and divergence were substantial $(80 \mathrm{~ms}$ for vergence movements between $20 \mathrm{~cm}$ and $40 \mathrm{~cm}$, and $36 \mathrm{~ms}$ for vergence movements between $40 \mathrm{~cm}$ and $150 \mathrm{~cm}$ ). Such increased latency for convergence in elderly is consistent with an EEG study (Tzelepi, Lutz \& Kapoula, 2004) showing massive central and posterior cortical activation prior to convergence movements in young subjects.

In conclusion, this study indicates differential aging effects on vergence: slowing of regular type of latency, particularly for convergence, and good preservation of express type of latency.

\section{Acknowledgement}

Q.Yang was supported by European Union (QLK6CT-2002-00151: EUROKINESIS) and CNRS/CTI, Handicap contract. 


\section{References}

Abel, L.A., Troost, B.T., \& Dell'Osso, L.F. (1983). The effects of age on normal saccadic characteristics and their variability. Vision Research, 23 (1), 33-37.

Alvarez, T.L., Semmlow, J.L., \& Pedrono, C. (2005). Divergence eye movements are dependent on initial stimulus position. Vision Research, 45 (14), 18471855 .

Brown, J.W., Bullock, D., \& Grossberg, S. (2004). How laminar frontal cortex and basal ganglia circuits interact to control planned and reactive saccades. Neural Networks, 17 (4), 471-510.

Bucci, M.P., Pouvreau, N., Yang, Q., \& Kapoula, Z. (2005). Influence of gap and overlap paradigms on saccade latencies and vergence eye movements in seven-year-old children. Experimental Brain Research, 164 (1), 48-57.

Carpenter, R. (1981). Oculomotor procrastination. In: D. Fischer, \& R. Monty (Eds.), Eye movements: cognition and visual perception. (pp. 237-246). Erlbaum: Hillsdale.

Chaturvedi, V., \& Van Gisbergen, J.A. (2000). Stimulation in the rostral pole of monkey superior colliculus: effects on vergence eye movements. Experimental Brain Research, 132 (1), 72-78.

Clarke, A.H., Ditterich, J., Druen, K., Schonfeld, U., \& Steineke, C. (2002). Using high frame rate CMOS sensors for three-dimensional eye tracking. Behav Res Methods Instrum Comput, 34 (4), 549-560.

Coubard, O., Daunys, G., \& Kapoula, Z. (2004). Gap effects on saccade and vergence latency. Experimental Brain Research, 154 (3), 368-381.

Coubard, O.A., \& Kapoula, Z. (2006). Dorsolateral prefrontal cortex prevents short-latency saccade and vergence: a TMS study. Cerebral Cortex 16 (3), 425436.

Creasey, H., \& Rapoport, S.I. (1985). The aging human brain. Annals of Neurology, 17 (1), 2-10.

Csibra, G., Johnson, M.H., \& Tucker, L.A. (1997). Attention and oculomotor control: a high-density ERP study of the gap effect. Neuropsychologia, 35 (6), 855-865. de Hemptinne, C., Lefevre, P., \& Missal, M. (2006). Influence of cognitive expectation on the initiation of anticipatory and visual pursuit eye movements in the rhesus monkey. Journal of Neurophysiology, 95 (6), 3770-3782.

Findlay, J.M., \& Walker, R. (1999). A model of saccade generation based on parallel processing and competitive inhibition. Behavioral and Brain Sciences, 22 (4), 661-674; discussion 674-721.

Fischer, B., \& Weber, H. (1993). Express saccades and visual attention. Behavioral and Brain Sciences, 16 . (3), 553-610.

Fischer, B., \& Weber, H. (1997). Effects of stimulus conditions on the performance of antisaccades in man. Experimental Brain Research, 116 (2), 191-200.

Fischer, B., Weber, H., Biscaldi, M., Aiple, F., Otto, P., \& Stuhr, V. (1993). Separate populations of visually guided saccades in humans: reaction times and amplitudes. Experimental Brain Research, 92 (3), 528-541.

Gamlin, P.D., \& Yoon, K. (2000). An area for vergence eye movement in primate frontal cortex. Nature, 407 (6807), 1003-1007.

Genovesio, A., \& Ferraina, S. (2004). Integration of retinal disparity and fixation-distance related signals toward an egocentric coding of distance in the posterior parietal cortex of primates. Journal of Neurophysiology, 91 (6), 2670-2684.

Gezeck, S., \& Timmer, J. (1998). Detecting multimodality in saccadic reaction time distributions in gap and overlap tasks. Biological Cybernetics, 78 (4), 293305 .

Goldring, J., \& Fischer, B. (1997). Reaction times of vertical prosaccades and antisaccades in gap and overlap tasks. Experimental Brain Research, 113 (1), 88-103.

Head, D., Buckner, R.L., Shimony, J.S., Williams, L.E., Akbudak, E., Conturo, T.E., McAvoy, M., Morris, J.C., \& Snyder, A.Z. (2004). Differential vulnerability of anterior white matter in nondemented aging with minimal acceleration in dementia of the Alzheimer type: evidence from diffusion tensor imaging. Cerebral Cortex 14 (4), 410-423.

Henson, C., Staunton, H., \& Brett, F.M. (2003). Does ageing have an effect on midbrain premotor nuclei for vertical eye movements? Movement disorder, 18 (6), 688-694. 
Huaman, A.G., \& Sharpe, J.A. (1993). Vertical saccades in senescence. Investigative Ophthalmology \& Visual Science 34 (8), 2588-2595.

Hung, G.K., Zhu, H., \& Ciuffreda, K.J. (1997). Convergence and divergence exhibit different response characteristics to symmetric stimuli. Vision Research, 37 (9), 1197-1205.

Irving, E.L., Steinbach, M.J., Lillakas, L., Babu, R.J., \& Hutchings, N. (2006). Horizontal saccade dynamics across the human life span. Investigative Ophthalmology \& Visual Science 47 (6), 2478-2484.

Isa, T., \& Kobayashi, Y. (2004). Switching between cortical and subcortical sensorimotor pathways. Progress in Brain Research, 143, 299-305.

Kapoula, Z., Isotalo, E., Muri, R.M., Bucci, M.P., \& Rivaud-Pechoux, S. (2001). Effects of transcranial magnetic stimulation of the posterior parietal cortex on saccades and vergence. Neuroreport, 12 (18), 40414046.

Kapoula, Z., Yang, Q., Coubard, O., Daunys, G., \& Orssaud, C. (2005a). Contextual influence of TMS on the latency of saccades and vergence. Neuroscience Letters, 376 (2), 87-92.

Kapoula, Z., Yang, Q., Coubard, O., Daunys, G., \& Orssaud, C. (2005b). Role of the posterior parietal cortex in the initiation of saccades and vergence: right/left functional asymmetry. Annals of the New York Academy of Sciences, 1039, 184-197.

Katzman, R., \& Terry, R. (1983). The neurology of aging. Philadelphia: F.A. Davis Co.

Kingstone, A., \& Klein, R.M. (1993). Visual offsets facilitate saccadic latency: does predisengagement of visuospatial attention mediate this gap effect? Journal of Experimental Psychology Human Perception and Performance, 19 (6), 1251-1265.

Knox, P.C., Davidson, J.H., \& Anderson, D. (2005). Agerelated changes in smooth pursuit initiation. Experimental Brain Research, 165 (1), 1-7.

Krishnan, V.V., Farazian, F., \& Stark, L. (1973). An analysis of latencies and prediction in the fusional vergence system. American Journal of Optometry \& Archives of American Academy of Optometry, 50 (12), 933-939.
Kumar, A.N., Han, Y., Garbutt, S., \& Leigh, R.J. (2002). Properties of anticipatory vergence responses. Investigative Ophthalmology \& Visual Science 43 (8), 2626-2632.

Leigh, R.J., \& Zee, D.S. (2006). The Neurology of Eye Movements. New York: Oxford University Press.

Mays, L.E., Porter, J.D., Gamlin, P.D., \& Tello, C.A. (1986). Neural control of vergence eye movements: neurons encoding vergence velocity. Journal of $\mathrm{Neu}$ rophysiology, 56 (4), 1007-1021.

Munoz, D.P., Broughton, J.R., Goldring, J.E., \& Armstrong, I.T. (1998). Age-related performance of human subjects on saccadic eye movement tasks. Experimental Brain Research, 121 (4), 391-400.

Muri, R.M., Vermersch, A.I., Rivaud, S., Gaymard, B., \& Pierrot-Deseilligny, C. (1996). Effects of single-pulse transcranial magnetic stimulation over the prefrontal and posterior parietal cortices during memory-guided saccades in humans. Journal of Neurophysiology, 76 (3), 2102-2106.

Ohtsuka, K., Maeda, S., \& Oguri, N. (2002). Accommodation and convergence palsy caused by lesions in the bilateral rostral superior colliculus. American Journal of Ophthalmology, 133 (3), 425-427.

Pierrot-Deseilligny, C., Muri, R.M., Ploner, C.J., Gaymard, B., \& Rivaud-Pechoux, S. (2003). Cortical control of ocular saccades in humans: a model for motricity. Progress in Brain Research, 142, 3-17.

Rambold, H., Neumann, G., Sander, T., \& Helmchen, C. (2006). Age-related changes of vergence under natural viewing conditions. Neurobiology of Aging, 27 (1), 163-172.

Rashbass, C., \& Westheimer, G. (1961). Disjunctive eye movements. Journal of Physiology 159, 339-360.

Reuter-Lorenz, P.A., Hughes, H.C., \& Fendrich, R. (1991). The reduction of saccadic latency by prior offset of the fixation point: an analysis of the gap effect. Perception \& Psychophysics, 49 (2), 167-175.

Rosen, R. (1978). Feedforwards and global system failure: a general mechanism for senescence. Journal of Theoretical Biology, 74 (4), 579-590.

Ross, L.E., \& Ross, S.M. (1980). Saccade latency and warning signals: stimulus onset, offset, and change as warning events. Perception \& Psychophysics, 27 (3), 251-257. 
Ross, S.M., \& Ross, L.E. (1981). Saccade latency and warning signals: effects of auditory and visual stimulus onset and offset. Perception \& Psychophysics, 29 (5), 429-437.

Rossini, P.M., Rossi, S., Babiloni, C., \& Polich, J. (2007). Clinical neurophysiology of aging brain: From normal aging to neurodegeneration. Progress in Neurobiology, 83 (6), 375-400.

Salat, D.H., Kaye, J.A., \& Janowsky, J.S. (2001). Selective preservation and degeneration within the prefrontal cortex in aging and Alzheimer disease. Archives of Neurology, 58 (9), 1403-1408.

Saslow, M.G. (1967). Effects of components of displacement-step stimuli upon latency for saccadic eye movement. Journal of the Optical Society of America 57 (8), 1024-1029.

Schiller, P.H., Sandell, J.H., \& Maunsell, J.H. (1987). The effect of frontal eye field and superior colliculus lesions on saccadic latencies in the rhesus monkey. Journal of Neurophysiology, 57 (4), 1033-1049.

Schiller, P.H., \& Tehovnik, E.J. (2005). Neural mechanisms underlying target selection with saccadic eye movements. Progress in Brain Research, 149, 157171.

Semmlow, J., \& Wetzel, P. (1979). Dynamic contributions of the components of binocular vergence. Journal of the Optical Society of America 69 (5), 639645.

Suzuki, S., Suzuki, Y., \& Ohtsuka, K. (2004). Convergence eye movements evoked by microstimulation of the rostral superior colliculus in the cat. Neuroscience Research, 49 (1), 39-45.

Takagi, M., Frohman, E.M., \& Zee, D.S. (1995). Gapoverlap effects on latencies of saccades, vergence and combined vergence-saccades in humans. Vision Research, 35 (23-24), 3373-3388.

Tam, W.J., \& Ono, H. (1994). Fixation disengagement and eye-movement latency. Perception \& Psychophysics, 56 (3), 251-260.

Tzelepi, A., Lutz, A., \& Kapoula, Z. (2004). EEG activity related to preparation and suppression of eye movements in three-dimensional space. Experimental Brain Research, 155 (4), 439-449.
Vijayashankar, N., \& Brody, H. (1977a). Aging in the human brain stem. A study of the nucleus of the trochlear nerve. Acta Anat (Basel), 99 (2), 169-172.

Vijayashankar, N., \& Brody, H. (1977b). A study of aging in the human abducens nucleus. Journal of Comparative Neurology, 173 (3), 433-438.

Weber, H., \& Fischer, B. (1995). Gap duration and location of attention focus modulate the occurrence of left/right asymmetries in the saccadic reaction times of human subjects. Vision Research, 35 (7), 987-998.

Yang, Q., Bucci, M.P., \& Kapoula, Z. (2002). The latency of saccades, vergence, and combined eye movements in children and in adults. Investigative Ophthalmology \& Visual Science 43 (9), 2939-2949.

Yang, Q., \& Kapoula, Z. (2004). TMS over the left posterior parietal cortex prolongs latency of contralateral saccades and convergence. Investigative Ophthalmology \& Visual Science 45 (7), 2231-2239.

Yang, Q., \& Kapoula, Z. (2006). The control of vertical saccades in aged subjects. Experimental Brain Research, 171 (1), 67-77.

Yang, Q., Kapoula, Z., Debay, E., Coubard, O., Orssaud, C., \& Samson, M. (2006). Prolongation of latency of horizontal saccades in elderly is distance and task specific. Vision Research, 46 (5), 751-759. 